Bull. Korean Math. Soc. 47 (2010), No. 4, pp. 663-673

DOI 10.4134/BKMS.2010.47.4.663

\title{
MODULE EXTENSION OF DUAL BANACH ALGEBRAS
}

\author{
Madjid Eshaghi Gordji, Fereydoun Habibian, and Ali Rejali
}

ABstract. This work was intended as an attempt to introduce and investigate the Connes-amenability of module extension of dual Banach algebras. It is natural to try to study the weak $k^{*}$-continuous derivations on the module extension of dual Banach algebras and also the weak Connesamenability of such Banach algebras.

\section{Introduction}

In [6], B. E. Johnson introduced the notion of an amenable Banach algebra, and proved that a locally compact group $G$ is amenable if and only if its group algebra $L^{1}(G)$ is amenable. The theory of amenable Banach algebras has been a very active field of research ever since. Once of the deepest result in this theory is due to Connes [2] and A. Haagerup [5]: a $C^{*}$-algebra is amenable if and only if it is nuclear. In [11], S. Wassermann showed that a von Neumann algebra is nuclear/amenable if and only if it is subhomogenuous [8]. This suggests that the definition of amenability from [6] has to be modified to yield a sufficiently rich theory for von Neumann algebras. A variant of that definition, one that takes the dual space structure of von Neumann algebra into account, was introduced in [7], but is most commonly associated with A. Connes paper [1]. For this reason, we refer to this notion of amenability as Connes-amenability. The definition of Connes-amenability makes sense for large class of Banach algebras (called dual Banach algebras in [8]). Examples of dual Banach algebras are: $B(\mathrm{E})$, where $E$ is a reflexive Banach space; $M(G)$, where $G$ is a locally compact group; $\mathfrak{A}^{* *}$, where $\mathfrak{A}$ is an Arens regular Banach algebra.

This paper is organized as follows. Section 1 is devoted to the notations and definitions which are needed throughout of the paper. The Connes-amenability of module extension of dual Banach algebras is studied in Section 2. Finally in Section 3, we investigate the weak Connes-amenability of module extension of dual Banach algebras.

Received December 8, 2008.

2000 Mathematics Subject Classification. 46H20, 43A20.

Key words and phrases. derivation, Connes-amenable. 


\section{Preliminaries}

This section is preliminary in character. For a Banach algebra $\mathfrak{A}$, an $\mathfrak{A}$ bimodule will always refer to a Banach $\mathfrak{A}$-bimodule $X$, that is a Banach space which is algebraically an $\mathfrak{A}$-bimodule, and for which there is a constant $C_{\mathfrak{A}, X}>$ 0 such that

$$
\|a . x\|,\|x . a\| \leq C_{\mathfrak{A}, X}\|a\|\|x\| \quad(a \in \mathfrak{A}, x \in X) .
$$

Let $\mathfrak{A}$ be a Banach algebra and $X$ be a Banach $\mathfrak{A}$-bimodule. Then $X^{*}$ is a Banach $\mathfrak{A}$-bimodule by the operations,

$$
\left\langle a x^{*}, x\right\rangle=\left\langle x^{*}, x a\right\rangle, \quad\left\langle x^{*} a, x\right\rangle=\left\langle x^{*}, a x\right\rangle,
$$

where $a \in \mathfrak{A}, x \in X$ and $x^{*} \in X^{*}$.

Definition 1.1. Let $\mathfrak{A}$ be a Banach algebra. A Banach $\mathfrak{A}$-bimodule $E$ is called dual if there is a closed submodule $E_{*}$ of $E^{*}$ such that $E=\left(E_{*}\right)^{*}$. $E_{*}$ is called the predual of $E$. A Banach algebra $\mathfrak{A}$ is called dual if it is dual as a Banach A-bimodule.

Let $\mathfrak{A}$ be a dual Banach algebra. A dual Banach $\mathfrak{A}$-bimodule $E$ is called normal if, for every $x \in E$, the maps

$$
\mathfrak{A} \longrightarrow E, \quad a \mapsto a . x
$$

and

$$
\mathfrak{A} \longrightarrow E, \quad a \mapsto x . a
$$

are weak ${ }^{*}$-continuous.

Let $\mathfrak{A}$ and $\mathfrak{B}$ be dual Banach algebras and let $\varphi: \mathfrak{A} \longrightarrow \mathfrak{B}$ be a weak ${ }^{*}$ continuous Banach algebra homomorphism. Then $\mathfrak{B}$ is a normal $\mathfrak{A}$-bimodule by the following module actions

$$
a . b=\varphi(a) b, \quad b . a=b \varphi(a) \quad(a \in \mathfrak{A}, b \in \mathfrak{B}) .
$$

We denote the above $\mathfrak{A}$-bimodule by $\mathfrak{B}_{\varphi}$. Let $X$ be a Banach $\mathfrak{A}$-bimodule. A derivation from $\mathfrak{A}$ into an $\mathfrak{A}$-bimodule $X$ is a bounded linear map $D$ such that $D(a b)=D(a) \cdot b+a \cdot D(b)$ for all $(a, b \in \mathfrak{A})$. If $x \in X$, then $\delta_{x}: \mathfrak{A} \longrightarrow X$ defined by

$$
\delta_{x}(a)=a . x-x . a \quad(a \in \mathfrak{A}),
$$

is a derivation. Such derivations are called inner. A Banach algebra $\mathfrak{A}$ is amenable if, for every $\mathfrak{A}$-bimodule $X$, every derivation $D: \mathfrak{A} \longrightarrow X^{*}$ is inner, equivalently if $H^{1}\left(\mathfrak{A}, X^{*}\right)=\{0\}$ for every Banach $\mathfrak{A}$-bimodule $X$, where the quotient space $H^{1}\left(\mathfrak{A}, X^{*}\right)$ of all continuous derivations from $\mathfrak{A}$ into $X^{*}$ modulo the subspace of all inner derivations from $\mathfrak{A}$ into $X^{*}$ is called the first cohomology group with coefficients in $X^{*}[3]$ (see [1] and [5] for more details).

Let $\mathfrak{A}$ be a dual Banach algebra. $\mathfrak{A}$ is called Connes-amenable if, for every dual Banach $\mathfrak{A}$-bimodule $X$, every weak $k^{*}$-continuous derivation $D: \mathfrak{A} \longrightarrow X$ is inner; or equivalently, $H_{w^{*}}^{1}(\mathfrak{A}, X)=\{0\}$. This definition was introduced by 
V. Runde (see Section 4 of [9]). A dual Banach algebra $\mathfrak{A}$ is weakly Connesamenable if every weak $k^{*}$-continuous derivation from $\mathfrak{A}$ into $\mathfrak{A}$ is inner; or equivalently, $H_{w^{*}}^{1}(\mathfrak{A}, \mathfrak{A})=\{0\}$ [4]. The weak amenability of module extension Banach algebras was studied by Y. Zhang in [12]. We define the module extensions of dual Banach algebras and then we study the Connes-amenability and the weak Connes-amenability of such Banach algebras.

\section{Connes-amenability}

In this section we give necessary and sufficient conditions for module extension of dual Banach algebras to be Connes-amenable.

Lemma 2.1. Let $\mathfrak{A}$ be a Banach algebra and $X$ be a Banach $\mathfrak{A}$-bimodule. $\mathfrak{A} \oplus_{\infty} X$ is a Banach algebra with the algebra product,

$$
(a, x)(b, y)=(a b, a y+x b)
$$

and with the norm,

$$
\|(a, x)\|=\max \{\|x\|,\|a\|\} \quad(a \in \mathfrak{A}, x \in X) .
$$

Proof. It is easily seen that $\mathfrak{A} \oplus_{\infty} X$ is a Banach space. But $\mathfrak{A}$ is a Banach algebra, then there exists $C_{\mathfrak{A}}>0$ such that $\|a b\| \leq C_{\mathfrak{A}}\|a\|\|b\|$ for all $a, b \in \mathfrak{A}$ (see page 152 of [3] for more details). Also there is a constant $C_{\mathfrak{A}, X}>0$ such that

$$
\|a . x\|,\|x . a\| \leq C_{\mathfrak{A}, X}\|a\|\|x\| \quad(a \in \mathfrak{A}, x \in X) .
$$

Fix $(a, x),(b, y) \in \mathfrak{A} \oplus_{\infty} X$. The proof falls naturally into four cases, but we give the proof for one case, the other cases are similar. If $\|(a, x)\|=$ $\operatorname{Max}\{\|x\|,\|a\|\}=\|a\|$ and $\|(b, y)\|=\operatorname{Max}\{\|y\|,\|b\|\}=\|b\|$, then

$$
\|a y+x b\| \leq\|a y\|+\|x b\| \leq C_{\mathfrak{A}, X}\|a\|\|y\|+C_{\mathfrak{A}, X}\|x\|\|b\| \leq 2 C_{\mathfrak{A}, X}\|a\|\|b\| .
$$

Set $C=\operatorname{Max}\left\{C_{\mathfrak{A}}, 2 C_{\mathfrak{A}, X}\right\}$. Now, if $\operatorname{Max}\{\|a b\|,\|a y+x b\|\}=\|a b\|$, then

$$
\begin{aligned}
\|(a, x)(b, y)\|=\|(a b, a y+x b)\| & =\|a b\| \leq C_{\mathfrak{A}}\|a\|\|b\| \\
& =C_{\mathfrak{A}}\|(a, x)\|\|(b, y)\| \\
& \leq C\|(a, x)\|\|(b, y)\| .
\end{aligned}
$$

If $\operatorname{Max}\{\|a b\|,\|a y+x b\|\}=\|a y+x b\|$, then

$$
\begin{aligned}
\|(a, x)(b, y)\| & =\|(a b, a y+x b)\|=\|a y+x b\| \\
& \leq 2 C_{\mathfrak{A}, X}\|a\|\|b\| \leq C\|a\|\|b\| \\
& =C\|(a, x)\|\|(b, y)\| .
\end{aligned}
$$

Then $\left(\mathfrak{A} \oplus_{\infty} X,\|\cdot\|\right)$ is a Banach algebra. Also if we define a new norm on $\mathfrak{A} \oplus_{\infty} X$, say, $\|\mid(a, x)\|\|=C\|(a, x) \|$, then $\left(\mathfrak{A} \oplus_{\infty} X,\||\cdot \||)\right.$ is a Banach algebra with

$$
\||(a, x)(b, y)\||\leq\||(a, x)\||\||(b, y) \|| .
$$


We define a new class of dual Banach algebras. Let $\mathfrak{A}$ be a dual Banach algebra with predual $\mathfrak{A}_{*}$, and let $X$ be a normal dual Banach $\mathfrak{A}$-bimodule with predual $X_{*}$.

It is a simple matter to check that $\mathfrak{A}_{*} \oplus_{1} X_{*}$ is a Banach space with the norm

$$
\left\|\left(a^{\prime}, x^{\prime}\right)\right\|=\left\|a^{\prime}\right\|+\left\|x^{\prime}\right\| \quad\left(a^{\prime} \in \mathfrak{A}_{*}, x^{\prime} \in X_{*}\right) .
$$

and $\mathfrak{A} \oplus_{\infty} X=\left(\mathfrak{A}_{*} \oplus_{1} X_{*}\right)^{*}$. Since $\mathfrak{A}$ is a dual Banach algebra and $X$ is a normal dual Banach $\mathfrak{A}$-bimodule, then clearly the multiplication in $\mathfrak{A} \oplus_{\infty} X$ is separately weak ${ }^{*}$-continuous. According to Exercise 4.4.1 of [9], we get the following lemma.

Lemma 2.2. Let $\mathfrak{A}$ and $X$ be as above. Then $\mathfrak{A} \oplus_{\infty} X$ is a dual Banach algebra with predual $\mathfrak{A}_{*} \oplus_{1} X_{*}$.

The Banach algebra $\mathfrak{A} \oplus_{\infty} X$ in Lemma 2.2, is said to be the module extension of dual Banach algebras.

Theorem 2.3. Let $\mathfrak{A}$ be a dual Banach algebra. Then the following assertions are equivalent:

(i) $\mathfrak{A}$ is Connes-amenable.

(ii) For every dual Banach algebra $\mathfrak{B}$ and every weak $k^{*}$-continuous homomorphism $\varphi: \mathfrak{A} \longrightarrow \mathfrak{B}, H_{w^{*}}^{1}\left(\mathfrak{A}, \mathfrak{B}_{\varphi}\right)=\{0\}$.

(iii) For every dual Banach algebra $\mathfrak{B}$ and every injective weak ${ }^{*}$-continuous homomorphism $\varphi: \mathfrak{A} \longrightarrow \mathfrak{B}, H_{w^{*}}^{1}\left(\mathfrak{A}, \mathfrak{B}_{\varphi}\right)=\{0\}$.

Proof. It is straightforward to verify (i) $\Rightarrow$ (ii) $\Rightarrow$ (iii). We prove (iii) $\Rightarrow$ (i). Let $X$ be a normal dual Banach $\mathfrak{A}$-bimodule, and let $D: \mathfrak{A} \longrightarrow X$ be a weak ${ }^{*}$ continuous derivation. Lemma 2.2 shows that the map

$$
\varphi: a \mapsto(a, 0), \quad \mathfrak{A} \longrightarrow \mathfrak{A} \oplus_{\infty} X
$$

is an injective weak $k^{*}$-continuous homomorphism. Hence $H_{w^{*}}^{1}\left(\mathfrak{A},\left(\left(\mathfrak{A} \oplus_{\infty} X\right)_{\varphi}\right)\right)$ $=\{0\}$. We define $D_{1}: \mathfrak{A} \longrightarrow \mathfrak{A} \oplus_{\infty} X$ by $D_{1}(a)=(0, D(a))$. For each $a, b \in \mathfrak{A}$,

$$
\begin{aligned}
D_{1}(a b) & =(0, D(a b))=(0, D(a) b+a D(b)) \\
& =(0, D(a))(b, 0)+(a, 0)(0, D(b)) \\
& =D_{1}(a) \varphi(b)+\varphi(a) D_{1}(b) .
\end{aligned}
$$

Therefore $D_{1}$ is a weak ${ }^{*}$-continuous derivation from $\mathfrak{A}$ into $\left(\mathfrak{A} \oplus_{\infty} X\right)_{\varphi}$. From this we conclude that $D_{1}$ is an inner derivation. On the other word, $D_{1}=\delta_{(b, x)}$ for some $b \in \mathfrak{A}, x \in X$. For every $a \in \mathfrak{A}$, we have

$$
\begin{aligned}
(0, D(a)) & =D_{1}(a)=\delta_{(b, x)}(a) \\
& =\varphi(a)(b, x)-(b, x) \varphi(a) \\
& =(a, 0)(b, x)-(b, x)(a, 0) \\
& =(a b-b a, a x-x a) .
\end{aligned}
$$

Hence $D=\delta_{x}$ and $\mathfrak{A}$ is Connes-amenable. 
We are thus led to give the main result.

Theorem 2.4. Let $\mathfrak{A}$ be a dual Banach algebra and let $X$ be a reflexive Banach $\mathfrak{A}$-bimodule. If for every $x^{\prime} \in X^{*}$ and $a \in \mathfrak{A}$, the mappings

(1) $\quad\left(x^{\prime} \widehat{\otimes} a\right) .: b \longmapsto\left(x^{\prime} \widehat{\otimes} a b\right), \quad .\left(x^{\prime} \widehat{\otimes} a\right): b \longmapsto\left(b x^{\prime} \widehat{\otimes} a\right) ; \quad \mathfrak{A} \longrightarrow X^{*} \widehat{\otimes} \mathfrak{A}$,

are weak ${ }^{*}$-weak continuous, then $\mathfrak{A} \oplus_{\infty} X$ is Connes-amenable if and only if $X=0$ and $\mathfrak{A}$ is Connes-amenable.

Proof. Let $\mathfrak{A} \oplus_{\infty} X$ be Connes-amenable and the mappings defined in (1), are weak ${ }^{*}$-weak continuous. We have to show that $X=0$. It is easy to check that $X^{*} \widehat{\otimes} \mathfrak{A}$ is a Banach $\mathfrak{A} \oplus_{\infty} X$-bimodule with the following module actions:

$\left(x^{\prime} \widehat{\otimes} a\right) .(b, x)=x^{\prime} \widehat{\otimes} a b,(b, x) .\left(x^{\prime} \widehat{\otimes} a\right)=b x^{\prime} \widehat{\otimes} a,\left(x^{\prime} \widehat{\otimes} a \in X^{*} \widehat{\otimes} \mathfrak{A},(b, x) \in \mathfrak{A} \oplus_{\infty} X\right)$.

Let

$$
\left(b_{\alpha}, x_{\alpha}\right) \stackrel{\text { weak }^{*}}{\longrightarrow}(b, x) \quad \text { in } \quad \mathfrak{A} \oplus_{\infty} X,
$$

hence $b_{\alpha} \stackrel{\text { weak }^{*}}{\longrightarrow} b$ in $\mathfrak{A}$. Then for each $x^{\prime} \in X^{*}$ and each $a \in \mathfrak{A}$,

$$
b_{\alpha} x^{\prime} \widehat{\otimes} a \stackrel{\text { weakly }}{\longrightarrow} b x^{\prime} \widehat{\otimes} a \quad \text { in } \quad X^{*} \widehat{\otimes} \mathfrak{A} .
$$

From this, for each $F \in\left(X^{*} \widehat{\otimes} \mathfrak{A}\right)^{*}$,

$$
\left\langle F .\left(b_{\alpha}, x_{\alpha}\right), x^{\prime} \widehat{\otimes} a\right\rangle=\left\langle F, b_{\alpha} x^{\prime} \widehat{\otimes} a\right\rangle \longrightarrow\left\langle F, b x^{\prime} \widehat{\otimes} a\right\rangle=\left\langle F .(b, x), x^{\prime} \widehat{\otimes} a\right\rangle .
$$

Consequently

$$
F .\left(b_{\alpha}, x_{\alpha}\right) \stackrel{\text { weak }^{*}}{\longrightarrow} F .(b, x) \quad \text { in }\left(X^{*} \widehat{\otimes} \mathfrak{A}\right)^{*} .
$$

Similarly

$$
\left(b_{\alpha}, x_{\alpha}\right) \cdot F \stackrel{\text { weak }^{*}}{\longrightarrow}(b, x) \cdot F \quad \text { in }\left(X^{*} \widehat{\otimes} \mathfrak{A}\right)^{*} .
$$

Thus $\left(X^{*} \widehat{\otimes} \mathfrak{A}\right)^{*}$ is a normal dual $\mathfrak{A} \oplus_{\infty} X$-bimodule. Define $D: \mathfrak{A} \oplus_{\infty} X \longrightarrow$ $\left(X^{*} \widehat{\otimes} \mathfrak{A}\right)^{*}$ by;

$$
\left\langle D(b, x), x^{\prime} \widehat{\otimes} a\right\rangle=\left\langle x^{\prime}, a x\right\rangle \quad\left(x^{\prime} \widehat{\otimes} a \in X^{*} \widehat{\otimes} \mathfrak{A},(b, x) \in \mathfrak{A} \oplus_{\infty} X\right) .
$$

For each $\left(b_{1}, x_{1}\right),\left(b_{2}, x_{2}\right) \in \mathfrak{A} \oplus_{\infty} X$, and $x^{\prime} \widehat{\otimes} a \in X^{*} \widehat{\otimes} \mathfrak{A}$, we have

$$
\begin{aligned}
& \left\langle D\left(\left(b_{1}, x_{1}\right)\left(b_{2}, x_{2}\right)\right), x^{\prime} \widehat{\otimes} a\right\rangle \\
= & \left\langle x^{\prime}, a\left(b_{1} x_{2}+x_{1} b_{2}\right)\right\rangle=\left\langle x^{\prime}, a b_{1} x_{2}\right\rangle+\left\langle x^{\prime}, a x_{1} b_{2}\right\rangle \\
= & \left\langle D\left(b_{2}, x_{2}\right), x^{\prime} \widehat{\otimes} a b_{1}\right\rangle+\left\langle D\left(b_{1}, x_{1}\right), b_{2} x^{\prime} \widehat{\otimes} a\right\rangle \\
= & \left\langle D\left(b_{2}, x_{2}\right),\left(x^{\prime} \widehat{\otimes} a\right)\left(b_{1}, x_{1}\right)\right\rangle+\left\langle D\left(b_{1}, x_{1}\right),\left(b_{2}, x_{2}\right)\left(x^{\prime} \widehat{\otimes} a\right)\right\rangle \\
= & \left\langle\left(b_{1}, x_{1}\right) \cdot\left(D\left(b_{2}, x_{2}\right)\right)+\left(D\left(b_{1}, x_{1}\right)\right) \cdot\left(b_{2}, x_{2}\right), x^{\prime} \widehat{\otimes} a\right\rangle .
\end{aligned}
$$

Hence $D$ is a derivation. Also if

$$
\left(b_{\alpha}, x_{\alpha}\right) \stackrel{\text { weak }^{*}}{\longrightarrow}(b, x) \quad \text { in } \mathfrak{A} \oplus_{\infty} X
$$


then $x_{\alpha} \stackrel{\text { weak }^{*}}{\longrightarrow} x$ in $X$. Since $X$ is a normal dual $\mathfrak{A}$-bimodule, we have $a x_{\alpha} \stackrel{w^{2} a k^{*}}{\longrightarrow}$ $a x$ in $X$. On the other hand, $X$ is reflexive, then $a x_{\alpha} \stackrel{\text { weakly }}{\longrightarrow} a x$ in $X$. Thus

$$
\left\langle D\left(b_{\alpha}, x_{\alpha}\right), x^{\prime} \widehat{\otimes} a\right\rangle=\left\langle x^{\prime}, a x_{\alpha}\right\rangle \longrightarrow\left\langle x^{\prime}, a x\right\rangle=\left\langle D(b, x), x^{\prime} \widehat{\otimes} a\right\rangle
$$

for every $x^{\prime} \widehat{\otimes} a \in X^{*} \widehat{\otimes} \mathfrak{A}$. Therefore $D$ is weak $k^{*}$-continuous. Connes-amenability of $\mathfrak{A} \oplus_{\infty} X$ implies that $D=\delta_{F}$ for some $F \in\left(X^{*} \widehat{\otimes} \mathfrak{A}\right)^{*}$. For each $x^{\prime} \widehat{\otimes} a \in X^{*} \widehat{\otimes} \mathfrak{A}$ and $(b, x) \in \mathfrak{A} \oplus_{\infty} X$, we have

$$
\begin{aligned}
\left\langle x^{\prime}, a x\right\rangle & =\left\langle D((b, x)), x^{\prime} \widehat{\otimes} a\right\rangle \\
& =\left\langle(b, x) \cdot F-F \cdot(b, x), x^{\prime} \widehat{\otimes} a\right\rangle \\
& =\left\langle F,\left(x^{\prime} \widehat{\otimes} a\right) \cdot(b, x)-(b, x)\left(x^{\prime} \widehat{\otimes} a\right)\right\rangle \\
& =\left\langle F, x^{\prime} \widehat{\otimes} a b-b x^{\prime} \widehat{\otimes} a\right\rangle .
\end{aligned}
$$

Then $\left\langle x^{\prime}, a x\right\rangle=0$ for each $a \in \mathfrak{A}, x \in X$ and $x^{\prime} \in X^{*}$. We have to show that $\mathfrak{A} X=X$. To this end, we know that if $\mathfrak{A} \oplus_{\infty} X$ is Connes-amenable, then it is unital [9]. Let $(e, x)$ be the unite element of $\mathfrak{A} \oplus_{\infty} X$. It is easy to show that $x=0$ and $e y=y$ for every $y \in X$, and this finishes the proof.

Corollary 2.5. Let $\mathfrak{A}$ be a dual Banach algebra and let $X$ be a non-trivial Banach $\mathfrak{A}$-bimodule. If $\mathfrak{A}$ and $X$ are reflexive, then $\mathfrak{A} \oplus_{\infty} X$ is not Connesamenable.

Corollary 2.6. Let $\mathfrak{A}$ be a non-trivial reflexive dual Banach algebra. Then the Banach algebras $\mathfrak{A} \oplus_{\infty} \mathfrak{A}$ and $\mathfrak{A} \oplus_{\infty} \mathfrak{A}^{*}$ are not Connes-amenable.

\section{Weak Connes-amenability}

Let $\mathfrak{A}$ be a dual Banach algebra with predual $\mathfrak{A}_{*}$, and let $X$ be a normal dual Banach $\mathfrak{A}$-bimodule with predual $X_{*}$. In this section we investigate the weak Connes-amenability of $\mathfrak{A} \oplus_{\infty} X$.

Lemma 3.1. Let $X$ be a normal, dual Banach $\mathfrak{A}$-bimodule and $T: X \longrightarrow X$ be a weak ${ }^{*}$-continuous $\mathfrak{A}$-bimodule morphism. Then $\bar{T}: \mathfrak{A} \oplus_{\infty} X \longrightarrow \mathfrak{A} \oplus_{\infty} X$, defined by $\bar{T}((a, x))=(0, T(x))$ is a weak ${ }^{*}$-continuous derivation. $\bar{T}$ is inner if and only if there exists $b \in \mathfrak{A}$ such that $b a=a b$ for each $a \in \mathfrak{A}$ and $T(x)=$ $x b-b x$ for all $x \in X$.

Proof. Let $(a, x),(b, y) \in \mathfrak{A} \oplus_{\infty} X$, we have

$\bar{T}((a, x) .(b, y))=\bar{T}((a b, a y+x b))=(0, T(a y+x b))=(0, a T(y))+(0, T(x) b)$.

On the other hand, $\bar{T}((a, x)) .(b, y)=(0, T(x)) .(b, y)=(0, T(x) b)$, similarly

$$
(a, x) \cdot \bar{T}((b, y))=(a, x) \cdot(0, T(x))=(0, a T(y)),
$$

and hence $\bar{T}$ is a derivation. From $w e a k^{*}$-continuity of $T$, it is clear that $\bar{T}$ is weak ${ }^{*}$-continuous. If $\bar{T}$ is inner, then there exists $\xi=(b, y) \in \mathfrak{A} \oplus_{\infty} X$ such that $\bar{T}((a, x))=(a, x) . \xi-\xi .(a, x)$. In particular $(0,0)=(a, 0) . \xi-\xi .(a, 0)$ and $(0, T(x))=(0, x) \cdot \xi-\xi \cdot(0, x)$. Then $(0,0)=(a b-b a, a y-y a)$ and $(0, T(x))=$ 
$(0, x b-b x)$ and so there exists $b \in \mathfrak{A}$ such that $b a=a b$ for $a \in \mathfrak{A}$ and $T(x)=x b-b x$ for all $x \in X$. Conversely, if there exists $b \in \mathfrak{A}$ such that $b a=a b$ for $a \in \mathfrak{A}$ and $T(x)=x b-b x$ for all $x \in X$, then

$$
\bar{T}((a, x))=(0, T(x))=(a b-b a, x b-b x)=(a, x) \cdot(b, 0)-(b, 0) \cdot(a, x) .
$$

Therefore $\bar{T}$ is inner.

Lemma 3.2. Let $\mathfrak{A}$ be a dual Banach algebra and let $X$ be a normal, dual Banach $\mathfrak{A}$-bimodule. If $D: \mathfrak{A} \longrightarrow X$ is a weak $k^{*}$-continuous derivation, then $\bar{D}:\left(\mathfrak{A} \oplus_{\infty} X\right) \longrightarrow\left(\mathfrak{A} \oplus_{\infty} X\right)$ defined by $\bar{D}((a, x))=(0, D(a))$, is a weak ${ }^{*}$ continuous derivation. Furthermore, $\bar{D}$ is inner if and only if $D$ is inner.

Proof. It is straightforward to check that $\bar{D}$ is a $w e a k^{*}$-continuous derivation. Let $\bar{D}$ be inner. Then there exists $\xi=(b, y) \in \mathfrak{A} \oplus_{\infty} X$ such that $\bar{D}((a, x))=$ $(a, x) . \xi-\xi .(a, x)$. In particular

$$
(0, D(a))=\bar{D}((a, 0))=(a, 0) \cdot(b, y)-(b, y) \cdot(a, 0)=(a b-b a, a y-y a),
$$

then $D(a)=a y-y a$ for some $y \in X$ and hence $D$ is inner. The same proof works for the converse.

Theorem 3.3. Let $\mathfrak{A}$ be a dual Banach algebra and let $X$ be a normal, dual Banach $\mathfrak{A}$-bimodule. Then $\mathfrak{A} \oplus_{\infty} X$ is weakly Connes-amenable if and only if the following conditions hold:

1. The only weak $k^{*}$-continuous derivations $D: \mathfrak{A} \longrightarrow \mathfrak{A}$ for which there is a weak $k^{*}$ continuous operator $T: X \longrightarrow X$ such that $T(a x)=D(a) x+a T(x)$ and $T(x a)=x D(a)+T(x) a(a \in \mathfrak{A}, x \in X)$, are the inner derivations.

2. $H_{w^{*}}^{1}(\mathfrak{A}, X)=\{0\}$.

3. The only weak ${ }^{*}$-continuous $\mathfrak{A}$-bimodule morphism $\Gamma: X \longrightarrow \mathfrak{A}$ for which $x \Gamma(y)+\Gamma(x) y=0(x, y \in X)$, is zero.

4. For every weak $k^{*}$-continuous $\mathfrak{A}$-bimodule morphism $T: X \longrightarrow X$, there exists $b \in \mathfrak{A}$ for which $a b=b a$ for $a \in \mathfrak{A}$ and $T(x)=x b-b x$ for $x \in X$.

Proof. Denote by $\tau_{1}$ and $\tau_{2}$ the inclusion mappings from, respectively, $\mathfrak{A}$ and $X$ into $\mathfrak{A} \oplus_{\infty} X$, and denote by $\Delta_{1}$ and $\Delta_{2}$ the natural projections from $\mathfrak{A} \oplus_{\infty} X$ onto $\mathfrak{A}$ and $X$, respectively. Then $\Delta_{1}$ and $\Delta_{2}$ are $\mathfrak{A}$-bimodule morphisms, so $\tau_{1}$ and $\tau_{2}$ are algebra homomorphisms. To prove the sufficiency we assume that Conditions 1-4 hold. Let $D: \mathfrak{A} \oplus_{\infty} X \longrightarrow \mathfrak{A} \oplus_{\infty} X$ be a weak ${ }^{*}$-continuous derivation. Then $\Delta_{1} \circ D \circ \tau_{1}: \mathfrak{A} \longrightarrow \mathfrak{A}$ and $\Delta_{2} \circ D \circ \tau_{1}: \mathfrak{A} \longrightarrow X$ are weak ${ }^{*}-$ continuous derivations. Now we show that $\Gamma=\Delta_{1} \circ D \circ \tau_{2}: X \longrightarrow \mathfrak{A}$ is trivial. By Condition 3 it suffices to show that $\Gamma$ is an $\mathfrak{A}$-bimodule morphism satisfying $x \Gamma(y)+\Gamma(x) y=0(x, y \in X)$. We have

$$
\begin{aligned}
0 & =D((0,0))=D((0, x) \cdot(0, y)) \\
& =D((0, x)) \cdot(0, y)+(0, x) \cdot D((0, y)) \\
& =(0, \Gamma(x) y)+(0, x \Gamma(y)) .
\end{aligned}
$$


On the other hand,

$$
\begin{aligned}
\Gamma(a x) & =\Delta_{1} \circ D((0, a x))=\Delta_{1} \circ D((a, 0) \cdot(0, x)) \\
& =\Delta_{1}(D((a, 0)) \cdot(0, x)+(a, 0) \cdot D((0, x))) \\
& =\Delta_{1}((a, 0) \cdot D((0, x)))=\Delta_{1}\left(a D \circ \tau_{2}(x)\right) \\
& =a \Gamma(x) .
\end{aligned}
$$

Similarly, $\Gamma(x a)=\Gamma(x) a$. Then $\Gamma$ is an $\mathfrak{A}$-bimodule morphism such that $x \Gamma(y)+\Gamma(x) y=0$. Therefore $\Gamma$ is trivial. Now let $T=\Delta_{2} \circ D \circ \tau_{2}: X \longrightarrow X$ and $D_{1}=\Delta_{1} \circ D \circ \tau_{1}: \mathfrak{A} \longrightarrow \mathfrak{A}$. For every $a \in \mathfrak{A}$ and $x \in X$,

$$
\begin{aligned}
(0, T(a x)) & =\left(0, \Delta_{2} \circ D((0, a x))=D((0, a x))\right. \\
& =D((a, 0) \cdot(0, x))=D((a, 0)) \cdot(0, x)+(a, 0) \cdot D((0, x)) \\
& =\left(0, D_{1}(a) x\right)+a(0, T(x))=\left(0, D_{1}(a) x+a T(x)\right) .
\end{aligned}
$$

This gives $T(a x)=D_{1}(a) x+a T(x)$. Similarly, for every $a \in \mathfrak{A}$ and $x \in X$, we have

$$
(0, T(x a))=\left(0, x D_{1}(a)+T(x) a\right) .
$$

Therefore by Condition $1, D_{1}=\Delta_{1} \circ D \circ \tau_{1}$ is inner.

Now suppose that $b \in \mathfrak{A}$ satisfies $D_{1}(a)=a b-b a$ for $a \in \mathfrak{A}$. Let $T_{1}: X \longrightarrow$ $X$ be defined by $T_{1}(x)=x b-b x$ for $x \in X$. Then $T-T_{1}: X \longrightarrow X$ is a weak ${ }^{*}$-continuous $\mathfrak{A}$-bimodule morphism. In fact, from (1), for every $a \in \mathfrak{A}$ and $x \in X$, we have

$$
\begin{aligned}
\left(T-T_{1}\right)(a x) & =T(a x)-T_{1}(a x) \\
& =\left(D_{1}(a) x+a T(x)\right)-(a x b-b a x) \\
& =(a b-b a) x+a T(x)-(a x b-b a x) \\
& =a(b x-x b)+a T(x)=a\left(T-T_{1}\right)(x) .
\end{aligned}
$$

Similarly, $T-T_{1}$ is a right $\mathfrak{A}$-bimodule morphism. From Condition 4 there is $c \in \mathfrak{A}$ such that $a c=c a$ for $a \in \mathfrak{A}$ and $\left(T-T_{1}\right)(x)=x c-c x$ for $x \in X$. By Lemma 3.1, we know that

$$
\overline{T-T_{1}}:(a, x) \longrightarrow\left(0,\left(T-T_{1}\right)(x)\right), \mathfrak{A} \oplus_{\infty} X \longrightarrow \mathfrak{A} \oplus_{\infty} X
$$

is an inner derivation. Since $\Delta_{2} \circ D \circ \tau_{1}: \mathfrak{A} \longrightarrow X$ is a weak $k^{*}$-continuous derivation, it is inner by Condition 2. By Lemma 3.2, the mapping

$$
\overline{\Delta_{2} \circ D \circ \tau_{1}}:(a, x) \longrightarrow\left(0, \Delta_{2} \circ D \circ \tau_{1}(a)\right), \mathfrak{A} \oplus_{\infty} X \longrightarrow \mathfrak{A} \oplus_{\infty} X
$$

is also an inner derivation. Since $\Gamma$ is trivial, we now have

$$
\begin{aligned}
D((a, x)) & =\left(D_{1}(a), \Delta_{2} \circ D \circ \tau_{1}(a)+T(x)\right) \\
& =\overline{\Delta_{2} \circ D \circ \tau_{1}}((a, x))+\overline{\left(T-T_{1}\right)}((a, x))+\left(D_{1}(a), T(x)\right) .
\end{aligned}
$$

Since

$$
\left(D_{1}(a), T_{1}(x)\right)=(a b-b a, x b-b x)=(a, x) \cdot(u, 0)-(u, 0) \cdot(a, x)
$$


for $a \in \mathfrak{A}$ and $x \in X$, it gives an inner derivation from $\mathfrak{A} \oplus_{\infty} X$ into $\mathfrak{A} \oplus_{\infty} X$. Hence as a sum of three inner derivations, $D$ is inner. According to Conditions $1-4, \mathfrak{A} \oplus_{\infty} X$ is weakly Connes-amenable.

Now we prove the necessity. Suppose that $\mathfrak{A} \oplus_{\infty} X$ is weakly Connesamenable. Let $D: \mathfrak{A} \longrightarrow \mathfrak{A}$ be a weak ${ }^{*}$-continuous derivation with the property given in Condition 1. We define $\bar{D}: \mathfrak{A} \oplus_{\infty} X \longrightarrow \mathfrak{A} \oplus_{\infty} X$ by

$$
\bar{D}((a, x))=(D(a), T(x)) \quad(a, x) \in\left(\mathfrak{A} \oplus_{\infty} X\right) .
$$

Then $\bar{D}$ is a weak $k^{*}$-continuous derivation. But $\bar{D}$ is inner, so there exists $(b, y) \in \mathfrak{A} \oplus_{\infty} X$ such that

$$
\bar{D}((a, x))=(a, x) \cdot(b, y)-(b, y) \cdot(a, x),
$$

and then for some $b \in \mathfrak{A}$, we have $(D(a), T(x))=(a b-b a, x b-b x)$. Thus $D(a)=$ $a b-b a$ for all $a \in \mathfrak{A}$, i.e., $D$ is inner, and Condition 1 holds. Condition 2 follows from Lemma 3.2. Let now $\Gamma: X \longrightarrow \mathfrak{A}$ be an arbitrary weak $k^{*}$-continuous $\mathfrak{A}$ bimodule morphism for which $x \Gamma(y)+\Gamma(x) y=0(x, y \in X)$. Define $\bar{\Gamma}$ : $\mathfrak{A} \oplus_{\infty} X \longrightarrow \mathfrak{A} \oplus_{\infty} X$ by $\bar{\Gamma}((a, x))=(\Gamma(x), 0)$ then $\bar{\Gamma}$ is a weak ${ }^{*}$-continuous derivation, but $\bar{\Gamma}$ is inner, hence there exists $\xi=(b, y) \in \mathfrak{A} \oplus_{\infty} X$ such that $\bar{\Gamma}((a, x))=(a, x) \cdot(b, y)-(b, y) \cdot(a, x)$. In particular

$$
(\Gamma(x), 0)=\bar{\Gamma}((0, x))=(0, x) \cdot(b, y)-(b, y) \cdot(0, x)=(0, x b-b x)
$$

and then $\Gamma=0$, and Condition 3 holds. Let $T: X \longrightarrow X$ be a weak ${ }^{*}$ continuous $\mathfrak{A}$-bimodule morphism. $\quad \bar{D}: \mathfrak{A} \oplus_{\infty} X \longrightarrow \mathfrak{A} \oplus_{\infty} X$ defined by $\bar{D}((a, x))=(0, T(x))$ is a weak $k^{*}$-continuous derivation, and Condition 4 holds by Lemma 3.1.

Let $X=\mathfrak{A}$. If in Condition 4 of above theorem, we suppose that $T=i d$ : $\mathfrak{A} \longrightarrow \mathfrak{A}$, thus we get:

Corollary 3.4. Let $\mathfrak{A}$ be a non-trivial dual Banach algebra $\mathfrak{A}$. Then,

$$
H_{w^{*}}^{1}\left(\mathfrak{A} \oplus_{\infty} \mathfrak{A}, \mathfrak{A} \oplus_{\infty} \mathfrak{A}\right) \neq\{0\} .
$$

Lemma 3.5. Let $X$ and $Y$ be dual Banach spaces. Then every weak ${ }^{*}$-continuous linear map from $X$ into $Y$ is bounded.

Proof. Let $T: X \longrightarrow Y$ be an unbounded linear map. Then there exists a sequence $\left\{x_{n}\right\}$ in $X$ such that $\lim _{n}\left\|x_{n}\right\|=0$ and $\lim _{n}\left\|T\left(x_{n}\right)\right\|=\infty$. By uniform boundedness theorem ([10]), $T\left(x_{n}\right) \stackrel{\text { weak }^{*}}{\longrightarrow} 0$. On the other hand weak*$\lim _{n} x_{n}=0$, therefore $T$ is not weak $k^{*}$-continuous.

Let us mention an important consequence of Corollary 3.4 and Lemma 3.5.

Corollary 3.6. Let $\mathfrak{A}$ be a non-trivial dual Banach algebra. Then

$$
H^{1}\left(\mathfrak{A} \oplus_{\infty} \mathfrak{A}, \mathfrak{A} \oplus_{\infty} \mathfrak{A}\right) \neq\{0\} .
$$


Let $\mathfrak{A}$ be a dual Banach algebra, and let $X=\mathfrak{A}$ by module actions

$$
a . x=a x, \quad x . a=0, \quad(a \in \mathfrak{A}, x \in X),
$$

We follow the notation of [12] to show that $X$ by $\mathfrak{A}_{0}$.

Corollary 3.7. $\mathfrak{A}$ is unital and weakly Connes-amenable if and only if $\mathfrak{A} \oplus_{\infty} \mathfrak{A}_{0}$ is weakly Connes-amenable.

Proof. Let $\mathfrak{A}$ be a unital weakly Connes-amenable Banach algebra. Since $\mathfrak{A}$ is weakly Connes-amenable, then the Conditions 1 and 2 in Theorem 3.3, hold. But $\mathfrak{A}$ is unital then Conditions 3 and 4 hold when $X=\mathfrak{A}_{0}$. For the converse let $\mathfrak{A} \oplus_{\infty} \mathfrak{A}_{0}$ be weakly Connes-amenable, then by Condition $2, \mathfrak{A}$ is weakly Connes-amenable. The mapping $i d: \mathfrak{A}_{0} \longrightarrow \mathfrak{A}_{0}$ is a weak $k^{*}$-continuous $\mathfrak{A}$ bimodule morphism, then by Condition 4 of Theorem 3.3, there exists $b \in \mathfrak{A}$ such that $a b=b a$ for $a \in \mathfrak{A}$, and $x=i d(x)=x . b-b . x=b x$ for $x \in \mathfrak{A}_{0}$. Thus $b$ is the unit element of $\mathfrak{A}$.

Acknowledgement. The first author would like to thank the Semnan University for its financial support. The third author would like to thank the center of Excellence of mathematics at Isfahan University for partially support of this research.

\section{References}

[1] A. Connes, Classification of injective factors. Cases $I I_{1}, I I_{\infty}, I I I_{\lambda}, \lambda \neq 1$, Ann. of Math. (2) 104 (1976), no. 1, 73-115.

[2] - On the cohomology of operator algebras, J. Functional Analysis 28 (1978), no. $2,248-253$.

[3] H. G. Dales, Banach Algebras and Automatic Continuity, London Mathematical Society Monographs. New Series, 24. Oxford Science Publications. The Clarendon Press, Oxford University Press, New York, 2000.

[4] M. Eshaghi Gordji, Left introverted subspaces of duals of Banach algebras and weak*continuous derivations on dual Banach algebras, Submitted.

[5] U. Haagerup, All nuclear $C^{*}$-algebras are amenable, Invent. Math. 74 (1983), no. 2, 305-319.

[6] B. E. Johnson, Cohomology in Banach Algebras, Memoirs of the American Mathematical Society, No. 127. American Mathematical Society, Providence, R.I., 1972.

[7] B. E. Johnson, R. V. Kadison, and J. Ringrose, Cohomology of operator algebras. III. Reduction to normal cohomology, Bull. Soc. Math. France 100 (1972), 73-96.

[8] V. Runde, Amenability for dual Banach algebras, Studia Math. 148 (2001), no. 1, 47-66.

[9] _ Lectures on Amenability, Springer-Verlage, Berlin, Hedinberg, New York, 2002.

[10] W. Rudin, Functional Analysis: second edition, Mcgraw-Hill, 1991.

[11] S. Wassermann, On tensor products of certain group $C^{*}$-algebras, J. Functional Analysis 23 (1976), no. 3, 239-254.

[12] Y. Zhang, Weak amenability of module extensions of Banach algebras, Trans. Amer. Math. Soc. 354 (2002), no. 10, 4131-4151. 


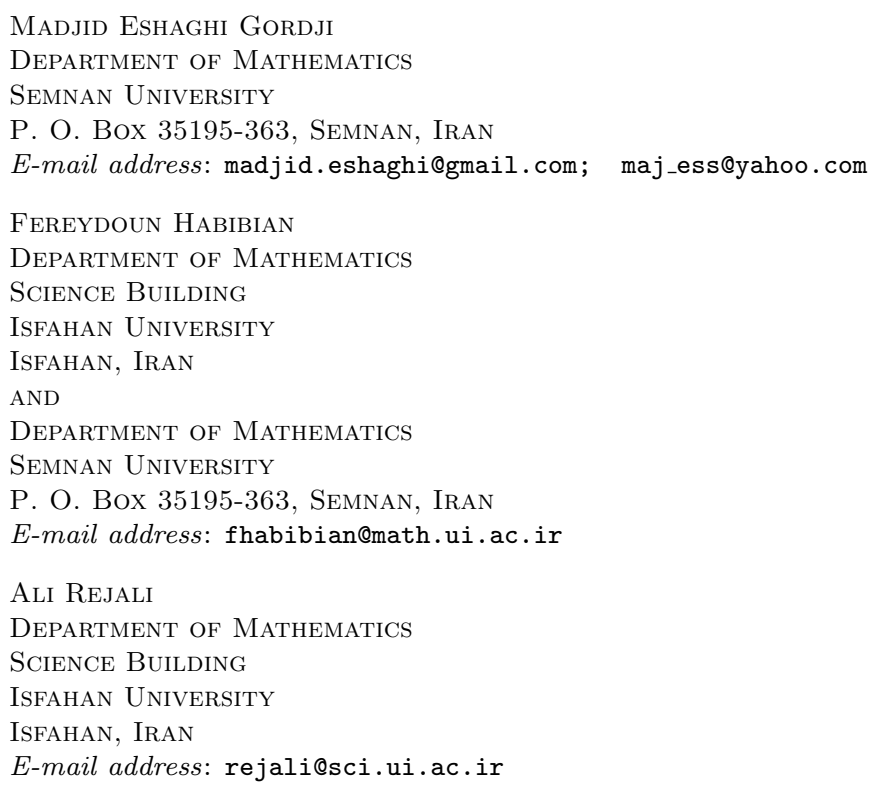

